\title{
Perspectives of Plasmapheresis in the Treatment of Kidney Diseases
}

\author{
Valerii Alexandrovich Voinov*, Konstantin Stepanovich Karchevsky, \\ Oleg Valerievich Isaulov
}

Gravitation Blood Surgery Department, I. P. Pavlov First Saint Petersburg State Medical University, Saint Petersburg, Russia

Email address:

voinof@mail.ru (V.A. Voinov), perfusiolog@mail.ru (K. S. Karchevsky), Isaulov1@gmail.com (O. V. Isaulov)

${ }^{*}$ Corresponding author

\section{To cite this article:}

Valerii Alexandrovich Voinov, Konstantin Stepanovich Karchevsky, Oleg Valerievich Isaulov. Perspectives of Plasmapheresis in the Treatment of Kidney Diseases. International Journal of Clinical Urology. Vol. 2, No. 1, 2018, pp. 6-14.

doi: $10.11648 /$ j.ijcu.20180201.12

Received: May 30, 2018; Accepted: June 22, 2018; Published: July 21, 2018

\begin{abstract}
Many kidney diseases pose a serious threat to human health and even life. In some cases, they inevitably lead to hemodialysis and even the need for kidney transplantation. Almost all chronic kidney disease in varying degrees, are associated with disorders of the internal environment, mainly autoimmune nature. Drug therapy is not always effective and can lead to additional disorders. Pathogenetically justified is the use of plasmapheresis. The aim of this study was to analyze the modern world literature in order to identify the features of the pathogenesis and course of various forms of chronic kidney disease in terms of determining the possibility of using plasmapheresis and indications for it. With the help of plasmapheresis, a number of problems can be solved in urology, in particular in the treatment of pyelonephritis, prostatitis and even male infertility.
\end{abstract}

Keywords: Kidney Diseases, Autoimmunity, Plasmapheresis, Male Infertility

\section{Introduction}

Almost all chronic kidney diseases in varying degrees are associated with disorders of the internal environment of the body, mainly autoimmune nature. Moreover, the mechanisms of these disorders are very diverse. Therefore, we have analyzed a variety of pathological conditions, but united on the principle of homeostasis disorders, which can be treated using apheresis technology, mainly plasmapheresis.

\section{Glomerulonephritis}

As well as rheumatism, glomerulonephritis also most often is serious complication of a streptococcal infection when toxins on the way of removal distort anti-gene structure of glomerular cells of kidneys and provoke of the autoantibodies formation which are fixed on a basal membrane of a nefron with are irreversible the progressing damage of the last $[1,2]$. Similarity of such succession of events to the aforesaid at rheumatism is confirmed by that in $25 \%$ of cases of glomerulonephritis really is combined with rheumatism. And the same as at rheumatism, here expediently perhaps earlier carrying out therapeutic apheresis - since the first signs of developing of this disease [3, 4], though often resort to a plasma exchange only at steroidresistant forms of a glomerulonephritis [5]. However steroid therapy is followed by many complications and in severe cases of its opportunity are limited [6]. The same can be told also about cytostatics. So, it was described development of an acute severe liver failure after reception cyclophosphamide for which treatment over required carrying out a plasma exchange [7].

There was described the development of rapidly progressive glomerulonephritis associated with the acute endocarditis caused by Streptococcus. With the help of plasmapheresis it was also able to restore renal function [8].

The retrospective analysis of results of treatment of children with idiopathic quickly progressing glomerulonephritis, and also a kidney or not kidney vasculitis, subjected to plasma exchange courses against the previous inefficient treatment by corticosteroids and cytostatics, showed considerable improvement of kidneys function at the majority of patients and a lasting positive effect at all patients from vasculitis $[9,10]$. At the same time 
the double-filt.ration plasmapheresis finds application also [11].

And, it was noted considerably much more big efficiency of a plasma exchange in comparison with hormonal therapy [12]. The same results were reached at 18 patients with quickly progressing glomerulonephritis against a systemic lupus erythematosis, a hemorrhagic vasculitis, Wegener's granulematosis and microscopic polyarteritis by means of courses from 5-10 procedures of a plasma exchange with removal a session of 1, 5-2 1 of plasma [13]. It allowed to stabilize or improve the course of kidney process and to increase survival of patients.

Quite often develops rather malignant course of glomerulonephritis with severe kidney damage, requiring dialysis therapy. The inclusion of plasmapheresis significantly faster leads to the restoration of renal function with the abolition of hemodialysis $[14,15]$.

Glomerulonephritis can arise and during a pregnancy and the plasma exchange against high doses of steroids allowed to stop in 3 months manifestations of an illness [16].

Of course, it is necessary to consider that glomerulonephritis, as well as any other autoimmune pathology, finally it is impossible to cure and systematic carrying out courses of a plasma exchange throughout all life is required. In cases of interruption of such treatment patients inevitably become hemodialysis-dependent or demand transplantation.

\subsection{Fibrillary Glomerulonephritis}

The fibrillary glomerulonephritis characterized by the deposition of non-amyloid fibrillary proteins (immune complexes) along globular membranes is the most frequent reason of an idiopathic nephrotic syndrome [17], often leading to development of a chronic renal failure, despite the carried-out immunosupressive therapy [18]. Carrying out three courses of a plasma exchange on 5-6 sessions each 3-5 months allowed to lower proteinuria degree from $7 \mathrm{~g}$ to $1 \mathrm{~g}$ a day [19].

\subsection{Membranoproliferative Glomerulonephritis the II Type}

It is a consequence of adjournment in a glomerular basement membrane of elements of a complement (Dense Deposite Disease) and meets most often at children. The course from 12 sessions of a plasma exchange with the subsequent purpose of an eculizumab provided permanent remission [20].

\subsection{Nephrotic Syndrome}

Inclusion of a plasma exchange at treatment of a glomerulonephritis against a nephrotic syndrome is especially indicated. It was applied thus equipment of a double-filtration plasmapheresis cycles on 3 sessions with introduction after the last Prednisolonum $(300 \mathrm{mg} / \mathrm{kg})$ and repetition of such courses monthly within half a year that allowed to reduce considerably degree of a proteinuria and permanently improve function of kidneys [21]. It was described a case when at treatment by steroids of a nephrotic syndrome at the patient who had hepatitis B the liver failure developed [22]. In the subsequent the cascade plasma exchange in the form of monotherapy to 9 sessions that led to decrease in level of a proteinuria from $9.2 \mathrm{~g}$ /day to $0.2 \mathrm{~g}$ /day was applied and such state kept and in 12 months. Also the combination of a cascade plasma exchange to immunoadsorption was effective [23].

Nevertheless, only timely removal of antibodies is capable to prevent or, at least, to reduce the scale of organic damage of a kidneys parenchyma. The same tactics is justified also at the subsequent aggravations, even at already arisen manifestations of a renal failure, for a distance of prospect of transition to a chronic hemodialysis.

Use of a plasma exchange of patients in a conservative phase of a chronic renal failure when the glomerular filtration is still kept at the level of $10-15 \mathrm{ml} /\left(\mathrm{min} \cdot \mathrm{m}^{2}\right)$ leads to fast improvement of health with lowering of arterial pressure, disappearance of an itch, decrease in level of creatinine and urea, increase in a glomerular filtration. In such cases the plasma exchange allowed to remove the moment of the transfer of patients to a chronic hemodialysis.

\section{Kidney Damage in Vasculitis}

The kidneys are the target organ in a wide variety of autoimmune diseases, particularly such as systemic lupus erythematosus (lupus nephritis), Goodpasture's syndrome, purpura Henoch-Schönlein, Wegener's granulomatosis, polyarteritis nodosa, in which vasculitis is an important component of the pathogenesis. And application of a plasma exchange is here too justified [24, 25].

\subsection{IgA Nephropathy}

IgA nephropathy or Berger's disease is the form of a glomerulonephritis, most widespread in the world, and, at the same time, the leading reason of a terminal renal failure [26]. Its etiology includes increase of production of polymeric A1 immunoglobulin with abnormal composition of some carbohydrate chains. The increasing quantity of the circulating immune complexes erise and settle in the renal mesangium with subsequent inflammatory activation of mesangial cells, segmental sclerosis and interstitial fibrosis/tubular atrophy [27, 28, 29]. Current therapy is not sufficiently effective. Therefore in resistance cases to treatment and quickly progressing current also the plasma exchange can be used [10, 30]. The IgA-nephropathy can be followed by pulmonary bleedings, and the plasma exchange also helps to cope them, including in children [31, 32]. IgAvasculitis is also characteristic of Henoch-Schönlein purpura, when glomerulonephritis can also develop like IgAnephropathy with hematuria and proteinuria up to terminal kidney damage.

\subsection{Thrombotic Microangiopathy}

The thrombotic microangiopathy (TMA) is a serious 
complication of the thrombotic thrombocytopenic purpura (TTP), as well as the hemolytic uremic syndrome (HUS) and hemolysis, elevated liver enzyme levels, low platelet count (HELLP), developing because of microvessels thrombosis associated with platelet thrombi [33, 34]. Thus system and kidney microvessels with development as anuries, and encephalopathies with damage and other vital organs. [35] The role and of a complement C5 activation is possible and the technique of release of a complement inhibitor monoclonal antibodies anti-C5 is now developed, though efficiency and intensive courses of a plasma exchange is shown [36, 37, 38, 39]. The case of a thrombotic microangiopathy with the phenomena of an acute renal failure against an antiphospholipid syndrome with a favorable outcome after a plasma exchange with immunosupression is described also [40]. A case of gemcitabine-induced TMA is also described that has also demanded application of a plasmapheresis [41].

\subsection{Periarteritis Nodosa}

The most common periarteritis nodosa, which is a generalized autoimmune necrotizing inflammation of the endothelium and the middle layer of medium and small arteries as a result of antigen-antibody reactions. Emerging granulomatosis while sometimes called "rash" at vessels. The clinical picture depends on the primary lesion of any organ of the abdominal or thoracic cavity, brain, limbs. Renal artery aneurysms with expanded with thrombosis and ruptures, leading to heart attacks and kidney hemorrhage. In another form of the disease - "microscopic" polyarteritis (Horton's disease) - affects mainly small arteries with the development of necrotizing glomerulonephritis. Perhaps the development also renal amyloidosis with proteinuria and renal insufficiency. In the treatment of this disease, along with steroid therapy was used the plasmapheresis also [42].

\subsection{ANCA-glomerulonephritis}

ANCA-glomerulonephritis develops as a consequence of vasculitis, when activated neutrophils penetrate through the walls of blood vessels and, releasing toxic oxygen radicals and destructive enzymes, undergo apoptosis and necrosis along with the lesion and vascular wall. This is accompanied by necrotic vasculitis [43]. Similar in pathogenesis of syndromes of Goodpasture and Wegener, in addition to the kidney, accompanied by the lung destruction and bleeding that requires connecting plasmapheresis [44].

\subsection{Diabetic Nephropathy}

Kidneys also suffer from diabetes - diabetic nephropathy is the leading cause of end-stage renal disease in the world [45]. The most common complication of diabetes is damage to the walls of microvessels by secondary toxic metabolites that accumulate despite even maintaining glucose levels at an acceptable level. The final products of glycation are one of the toxic factors determining the development of vascular complications in diabetes [46]. Among them we can mention peralin (pyraline) arising from the interaction of glucose with amino groups of proteins. In addition, the level of circulating proinflammatory cytokines contributing to the progression of kidney damage is increasing [47]. The accumulation of uric acid and glycated hemoglobin, as well as oxidative stress with increased activity of the renin-angiotensin-aldosterone system is important $[48,49]$. In this case, the thickening of the basal glomerular membrane, mesangial expansion, nodular glomerular sclerosis and tubulointerstitial fibrosis [50], immune disorders with infiltration of immune cells, mainly macrophages, and complement activation also play a role [51]. All this puts indications for the use of plasmapheresis, in particular, in diabetic ketoacidosis [52].

\subsection{Lupus Nephritis}

Lupus nephritis develops as a severe complication of SLE with a sufficiently large frequency. At the same time, circulating immune complexes are deposited in the renal vessels. Infiltration of renal parenchyma by T-lymphocytes, macrophages and dendritic cells occurs, which play a major role in the development of lupus glomerulonephritis leading to renal failure [53]. With the help of plasmapheresis it is possible to prevent the progression and fast enough to achieve remission [54]. It is possible to use immunoadsorption, which can be used to remove autoantibodies, but plasmapheresis removes activated complement components, clotting factors and cytokines [55]. The use of double-filtration plasmapheresis is also justified [56].

\section{Metabolic Disorders}

\subsection{Amyloidosis}

Kidney damage occurs in amyloidosis, when a clone of plasma cells secretes amyloidogenic light chains, which are deposited on the membranes of glomeruli, tubules and even microvessels. Severe nephrotic syndrome with proteinuria and progressive renal failure develops [57, 58, 59]. Moreover, amyloidosis can evolve on long-term hemodialysis, causing polyarthralgia and other organ lesions $[57,58]$.

\subsection{Multiple Myeloma}

Other types of monoclonal gammopathy, in particular multiple myeloma, carry the same risk to the kidneys [59]. The same can be said about cryoglobulinemia, leading to proteinuria, nephrotic syndrome and renal failure progression when plasmapheresis also helps to improve kidney function [60].

\section{Kidney Disorders at Infection Diseases}

\subsection{Hemorrhagic Fever to a Kidney Syndrome}

Kidneys suffer and at a number of infectious diseases. In particular, the combination of the hemorrhagic fever to a 
kidney syndrome caused the gantavirus is frequent. Thus the acute renal failure develops on the second week of an illness when IgM-antibodies to "not-Goodpastur's-antigene" of a glomerular membrane of kidneys come to light. Early use of plasmapheresis, including in children, can improve the outcome of the disease [4].

\subsection{Hemolytic Uremic Syndrome}

The hemolytic uremic syndrome (HUS) is characterized by a triad of thrombocytopenia, microangiopathic hemolytic anemia, and acute kidney damage [61]. It is the most common cause of acute kidney damage in children and is divided into 2 groups - typical with diarrhea (dHUS) and atypical, not associated with it (aHUS). The most common cause of a typical form is Shiga-toxin produced by Esherichia coli type O157:H7 [62]. In such case, severe enterocolitis occurs with the development of dHUS with renal failure and high mortality. Antibiotics were thus powerless or, on the contrary, contributed to the growth of endotoxicosis. This became clear by the end of such an epidemic in Europe in 2010-2011, which forced even to abandon antibiotic therapy. The use of plasmapheresis in the early stages of the disease contributed to the fast healing $[63,64,65]$ summarizing the experience of treatment of 631 patients in 84 hospitals in Germany, Sweden and the Netherlands, came to the same conclusion.

\subsection{Kidney Damage at Viral Hepatitis C}

Too most it is possible to tell and about viral hepatitis $\mathrm{C}$ at which the renal failure demanding a hemodialysis can develop too [66]. Hepatitis B can also lead to development of a secondary membranous nephropathy at which the plasma exchange renders medical effect $[67,68]$. Therefore, in all these cases, plasmapheresis is a pathogenetically justified method of treatment and prevention of progression of kidney lesions [60]. Indeed, the use of plasmapheresis in the presence of immunosuppressants allows for faster stabilization of renal function with the cessation of progression of renal failure [69]. At the same time, it is often necessary to remove very massive amounts of plasma - up to 2-2.5 1, at first 3 such sessions every other day, then 2-3 sessions every 2 weeks [70]. It is used cascade plasmapheresis also, promoting both removing viruses and reduction of HCV-associated cryoglobulin-nephropathy [71].

\section{Problems in Urology}

\subsection{Acute Pyelonephritis}

At acute pyelonephritis quite often there is a process of generalization, up to sepsis and multiple organ insufficiency to a serious endotoxicosis which can be stopped only by means of an intensive plasmapheresis or a plasma exchange with replacing by donor plasma [72].

Pyelonephritis, both at the height of exacerbation, and with a sluggish course with frequent relapses, is also not always amenable to drug and antibacterial therapy. All this makes it shown and apheresis therapy - hemosorption, plasmapheresis with laser irradiation of the blood. The use of plasmapheresis in acute purulent pyelonephritis accelerates recovery, reduces the frequency of nephrectomy by $14 \%$ [73]. Physiological immunosuppression during pregnancy contributes to the exacerbation of chronic pyelonephritis, which contributes to the appearance of preeclampsia with endotoxicosis. Courses of plasmapheresis with laser irradiation of blood stop such exacerbation and provide the best conditions for the development of the fetus [74].

\subsection{Chronic Pyelonephritis}

For chronic pyelonephritis is characteristic periodic coming activation of inflammatory process against both local weakening of the immune defence, and the general immunosuppression. Thus by means of a plasma exchange in combination with it is possible to achieve both a detoxification, and immunocorrection that phototherapy will help to warn or soften episodes of aggravations in the future [75]. This is because plasmapheresis removes the "toxic press" from the immune system, which helps to restore its activity.

\subsection{Prostatitis}

It is quite widespread disease and, nevertheless, having the long and hardly giving in to therapy current. Most often in its etiology urogenital chlamydiosis and mycoplasmosis matter. One of the promoting factors of chronization is the immunosuppressive state promoting such types of chronic infection. In such cases, with the help of plasmapheresis it is also possible to restore the immune response and stabilize such chronic inflammation.

\subsection{Interstitial Cystitis}

It is followed by pains and frequent desires on an urination perhaps has the autoimmune nature as about a half of these patients have the increased level of various autoantibodies [76]. It is impossible to exclude also allergic mechanisms, especially at a combination to allergic rhinitis and asthma [77]. Therefore the plasma exchange here too will be useful.

\subsection{Priapism}

In pathogenesis of such disease can play a role an antiprotein $\mathrm{S}$ antibodies leading to development of thrombosis in a cavernous body up to a necrosis of it and need of its removal. The plasma exchange is capable to prevent such complications [78].

\subsection{Male Infertility}

Among its reasons the essential role is played by autoimmune frustration when after quite often for a long time the forgotten injuries, an orchyepidimitis and even to the varicocele arise the autoantibodies against own spermatozoid which find in $8-21 \%$ of cases in men at suspicion on "man's" infertility [79]. They are capable to break both mobility of 
spermatozoa, and ability of the last to get through an oocyte cover without what extracorporeal fertilization which success in such cases doesn't exceed $40 \%$ is impossible even. There are indications that antisperm antibodies can be caused by the use of immunosuppressants and cytostatic agents, anti-parasitic medicines, prostate-specific antigen (PSA). Play a role and the presence of men Chlamydia trachomatis, Ureaplasma urealyticum and Microplasma hominis [80, 81].

In addition to autoantibodies, the functional state of spermatozoa can be influenced by other toxic compounds of both exo- and endogenous origin, in particular - the generation of excess amounts of cytotoxic oxidants [82, 83]. In such cases, the sperm cells lose the genes for the oxidative phosphorylation [84]. Thus the autoantibodies maintenance of such in blood can increase to 3000-4000 units/ml (in norm of 75 units $/ \mathrm{ml}$ ) and to $500-2000$ units $/ \mathrm{ml}$ in an ejaculate (at the same norm) [85]. It is described the antispermal autoantibodies emergence also at men with the revealed viral (HSV1, CMV) infections (31.1\%) or at their biocenosis from bacteria (61.4\%) [86]. With systemic lupus erythematosus, spermatogenesis with the appearance of autoantibodies is also disturbed and treatment is carried out with the help of cyclophosphamide [87].

Only by means of a plasma exchange it is possible to remove such antibodies and other toxic products out of an organism and to provide safe conditions for fertilization. So, it was noted increase of total of spermatozoa in an ejaculate for $46 \%$, and quantities of mobile cells for $30-123 \%$ after a plasma exchange course at men thus that in $42 \%$ of supervision at wives of these patients there came pregnancy [85]. Nevertheless even in U.S.A. insurance companies don't cover this cost for treatment of male infertility [88].

Our own brief experience also confirms these data.

Patient I, 45 years old. Several unsuccessful attempts were made to induce pregnancy by in vitro fertilization. In 2014, MAR-test could not be implemented due to the low concentration of motile sperm in the ejaculate (oligoasthenoteratozoospermia). 14.02.2015 - MAR-test $50 \% .27 .02-18.03 .2015$ it was held 4 sessions of membrane plasmapheresis. 19.03 - on spermogram - MAR-test - 0\%. It is obvious that these days, immediately after the course of plasmapheresis, the conception took place with the birth of a healthy child (10.12.2015). And after 2 years, this couple had a second independent conception.

\subsection{Decrease in a Potentiality}

Such problem can develop not only in old age, but also at younger ("reproductive") age. And deterioration of cavernous bodies blood filling as a result of the occlusion processes in the arteries can be one of the reasons. It arises most often against of system vessels damages at autoimmune vasculitis, the Buerger disease, the obliterating endarteritis and a diabetic vasculopathy. And the plasma exchange in such cases also helps to restore erectile function [89].

\section{Tactics of Treatment of Autoimmune Diseases}

The most common they are based on two-component drug therapy - corticosteroids and cytostatics. They are designed to delay reproduction of auto-reactive T-and B-lymphocytes clones. However, such therapy doesn't lack a large number of adverse reactions. Corticosteroids lead to cushingoid syndrome. Glucocorticoids, in particular, are diabetogenic hormones due to their suppression of glucose consumption by the tissues and increase of its production by the liver. Another complication of long-term glucocorticoid therapy is osteoporosis. It is believed that these hormones inhibit the proliferation and differentiation of osteoblasts and stimulate their apoptosis. There is also an indirect mechanism of bone resorption as a result of secondary hyperparathyroidism due to decreased calcium adsorption in the intestines.

Cytostatics lead to significant metabolic disorders, including healthy organs and systems. Intravenous administration of large doses of immunoglobulins are often used, leading to a significantly decreased content of pathological autoantibodies and inhibitors, and this effect exceeds the life of these immunoglobulins, which indicates a more significant regulatory correction of pathological autoimmune processes in the patients. However, intensive intravenous administration of immunoglobulins is reported to have a great number of complications.

The most common cause of patients' infection is said to be hepatitis G-viruses. Following such immunoglobulinotherapy, acute renal failure may develop. It also describes and serious complications such as vasculitis and lupus due to the side effects caused by immunoglobulins administered along with related autoantibodies and circulating immune complexes, lethal hypersensitive myocarditis, not to mention transmission of hepatitis viruses $\mathrm{C}$ and $\mathrm{D}$, etc. There is also a significant increase in blood viscosity associated with administration of high doses of immunoglobulins, which can create a number of problems in elderly patients, having vascular diseases, cryoglobulinemia, monoclonal gammopathy, and high levels of lipoproteins.

It should be taken into account that immunoglobulins are nothing but complexes of various antibodies. Among them there are not only useful antibodies against a variety of microbes and viruses, with which donors had to get into conflict during their lifetime, but also autoantibodies against their own structures antigens. And even if these donors are formally considered healthy, nevertheless, latent and not yet manifested symptoms of certain diseases are not excluded. In intensive immunoglobulins therapy immunoglobulin is simultaneously injected from many donors and the total number of certain autoantibodies can exceed the known critical mass and be manifested by additional pathology. This mass of immunoglobulins can turn from a "concentrate of health" into a "concentrate of diseases" [90].

In the recent years, treatment of autoimmune diseases with rituximab - chimeric monoclonal antibody to the CD20 antigen of B-lymphocytes - has also become widespread, 
which is meant to reduce the autoantibodies production. However, complications of such treatment are described, resulting even in development of multi-organ failure [91]. Rituximab, cetuximab and panitumumab have direct nephrotoxic effect [92]. Eculizumab can lead to a severe damage of kidneys, up to anury associated with haemolytic uraemic syndrome [93].

\section{Hemodialysis - Opportunities and Complications}

But even with a complete loss of kidney function, hemodialysis is not able to provide remove all pathological of compounds, particularly molecular weight of more than 1000 Daltons, ie, middle weight molecules, which do not pass through the dialysis membrane known, but cause a number of adverse metabolic disorders, among which more than just worried sick, itchy skin. In varying degrees, expressed it occurs in $60-90 \%$ of patients during hemodialysis. Its pathogenesis depends of many metabolic factors - hypercalcemia, hyperphosphatemia, secondary hyperparathyroidism, hypermagnesiemia, amyloidosis.

But even in the absence of metabolic disorders pruritus was significantly disturbed daily activity and nocturnal sleep [94].

In such cases, obviously, hemodialysis is simply not able enough to fully withdraw all products pathological metabolic and periodic addition of plasmapheresis can greatly improve the quality of life of these patients [95]. In particular, acute kidney failure with glomerulonephritis plasmapheresis in conjunction with hemodialysis facilitated more rapid relief of clinical symptoms and the achievement of stable remission. There is used also a "tandem method" simultaneous hemodialysis and plasmapheresis. At first the blood pass through the plasma filter, and then through the dialyzer [96].

In chronic hemodialysis often develop anemia, are not always amenable to erythropoietin administration and iron supplementation (Sorbifer). In such cases, periodic plasmapheresis helped stabilize hemoglobin levels and increased intervals between the infusions of packed red blood cells up to 6 months.

In essence, and any other form of renal parenchymal lesions (nephrosis or nephritis exo- or endotoxin genesis, lupus nephritis) therapeutic apheresis can prevent or reduce the disease occurred [97]. As it was already mentioned above, at a number of acute damages - poisonings, burns, severe injuries, a crush-syndrome, etc., timely removal of pathological products is capable to prevent organic damages of kidneys and will allow to avoid need of the subsequent hemodialyses.

Of course, not every acute renal pathology becomes chronic, but predict in advance such a situation is practically impossible, so even preventive perform in general completely

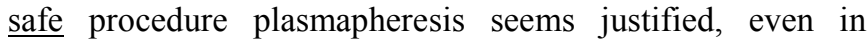
childhood. Especially when you consider that neither age nor body weight, have not additional restrictions for membrane plasmapheresis.

\section{Plasmapheresis}

This word comes from the Greek $\pi \lambda \alpha$ ó $\mu \alpha$ (liquid part of

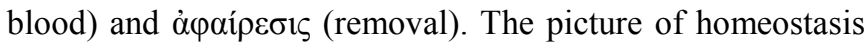
disorders, leading to organ disorders increase, increasingly becomes clearer. In this case, many pathological products are accumulating, the molecules size of which does not allow them to be eliminated by the kidneys while the liver is also unable to destroy them. On the other hand, the fact of their accumulation suggests that no drugs are able to help them to be removed from the body $[98,99]$.

Therefore in all these cases the plasmapheresis is pathogenetic justified method of treatment and prevention of progressing of kidneys damages [60]. And it is valid, use of a plasma exchange against immunodepressants allows to stabilize quicker function of kidneys with the termination of a renal failure progressing [60]. Thus, removal of very massive volumes of plasma - up to $2-2,51$ (1 CPV), in the beginning 3 such sessions every other day, then 2-3 sessions every 2 week often is required [13].

\section{Conclusion}

The provided findings show real dangers and serious complications of many kidney diseases, which not always can be prevented by drug therapy. This is because many large-molecular substances accumulate - antibodies, immune complexes and other pathological metabolites that cannot be excreted by the kidneys. On the other hand, the kidneys themselves are damaged due to their deposition in the microvessels, glomeruli, tubules, which causes an impairment of their functions, up to fatal disorders that require hemodialysis and even the need for transplantation of donor kidneys. Drug therapy itself can be dangerous due to serious side effects and not always provides a favorable outcome. Therefore, the question raises of physical removal of these accumulated pathological products together with the liquid part of the blood - plasma, which occurs in plasmapheresis. With the help of plasmapheresis, much better treatment results can be achieved, more stable remission with a lower level of drug support.

\section{References}

[1] Chandnani HK, Jain R, Patamasucon P. Group C streptococcus causing rheumatic heart disease in a child. J Emerg Med 2015; 49; 12-14.

[2] Fisher A. Die Wandlung der postinfektiösen Glomerulonephritis. Ther Umsch 2015; 72: 141-147.

[3] Haris A, Arányi J, Braunitzer $\mathrm{H}$ et al. Role of plasmapheresis in immunological kidney diseases. Experience from 1050 completed plasmapheresis treatment sessions. Orv Hetil 2011; 152 (28): 1110-1119.

[4] Reddy SK, Jahan A, Chaturvedi S, Agarwal I. Plasma exchange for paediatric kidney disease-indications and outcomes: a single-centre experience. Clin Kidney J 2015; 8 (6): 702-707. 
[5] Couzi L, Morel D, Deminière C, Merville P. An unusual endocarditis-induced crescentic glomerulonephritis treated by plasmapheresis. Clin Nephrol 2004; 62 (6): 461-464.

[6] Müller-Deile J, Schiffer M. A new rescue regimen with plasma exchange and rituximab in high-risk membranous glomerulonephritis. Eur J Clin Invest 2015; 45 (12): 12601269.

[7] Subramaniam SR, Cader RA, Mohd R et al. Low-dose cyclophosphamide-induced acute hepatotoxicity. Am J Case Rep 2013; 4 (14): 345-349.

[8] Halpin M, Kozyreva O, Bijol V, Jaber BL. Plasmapheresis for treatment of immune complex-mediated glomerulonephritis in infective endocarditis: a case report and literature review. Clin Nephrol Case Study 2017; 5: P. 26-31.

[9] Walsh M, Catapano F, Szpirt W et al. Plasma exchange for renal vasculitis and idiopathic rapidly progressive glomerulonephritis: meta-analysis. Am J Kidney Dis 2011; 57 (4): 566-574.

[10] Greenhall GH, Salama AD. What is new in the management of rapidly progressive glomerulonephritis? Clin Kidney J 2015; 8 (2): 143-150.

[11] Chen Y, Yang L, Li K et al. Double filtration plasmapheresis in the treatment of antineutrophil cytoplasmic autoantibody associated vasculitis with severe renal failure: a preliminare study of 15 patients. Ther Apher Dial 2016; 20 (2): 183-188.

[12] Jayne DR, Gaskin G, Rasmussen N et al. Randomized trial of plasma exchange or high-dosage methylprednisolone as adjunctive therapy for severe renal vasculitis. J Am Soc Nephrol 2007; 7: P. 2180-2188.

[13] Milovanov YuS, Komyagin YuV, Nikolaev AYu [Plasmapheresis in treatment of quickly progressing glomerulonephritis. Ther Arch (Rus) 1993; 6: 80-86.

[14] Fujigaki Y, Morimoto C, Ino R, Taniguchi K, Rawamotrita Y et al. Discontinuation of hemodialysis in a patient with antiGBM disease by the treatment with corticosteroids and plasmapheresis despite several predictors for dialysisdependence. Case Rep Nephrol 2017; 2017: 7143649.

[15] Usui T, Kai $H$, Noguchi $K$ et al. Effectiveness of plasmapheresis in a patient with anti-glomerular basement membrane antibody glomerulonephritis with advanced kidney dysfunction. Intern Med 2017; 56 (18): 2475-2479.

[16] Adnan MM, Morton J, Hashmi S et al. Anti-GBM disease in pregnancy: Acute renal failure resolved after plasma exchange, hemodialysis, and steroids. J Investig Med High Impact Case Rep 2015; 31 (4): 2314709615624232.

[17] Mallett A, Tang W, Hart G et al. End-stage kidney disease due to fibrillary glomerulonephritis and immunotactoid glomerulopathy - outcomes in 66 consecutive ANZDATA Registry Cases. Am J Nephrol 2015; 42 (3): 177-184.

[18] Cheungpasitporn W, Zacharek CC, Fervenza FC et al. Rapidly progressive glomerulonephritis due to coexistent antiglomerular basement membrane disease and fibrillary glomerulonephritis. Clin Lidney J 2016; 9 (1): 97-101.

[19] Pliquett RU, Mohr P, Mukhtar BD et al. Plasmapheresis leading to remission of refractory nephrotic syndrome due to fibrillary glomerulonephritis: a case report. J Med Case Rep 2012; (6): 116
[20] Nord AT, Nord BL, Schmidt AE, Smith DS. Management of Dense Deposit Disease with Plasmapheresis and eculizumab. J Clin Apher 2014; 29 (1): 28-29.

[21] Russo GE, Bonello M, Bauco B et al. Nephrotic syndrome and plasmapheresis. Int J Artif Organs 2000; 23 (2): 111-113.

[22] Kobayashi T, Ando Y, Umino T et al. Complete remission of minimal-change nephrotic syndrome induced by apheresis monotherapy. Clin Nephrol 2006; 65 (6): 423-426.

[23] Zhang YY, Tang Z, Chen DM et al. Comparison of double filtration plasmapheresis with immunoadsorbtion therapy in patients with anti-glomerular basement membrane nephritis. BMC Nephrol 2014; 15: 128.

[24] Clark W. F. Plasma exchange for renal disease: evidence and use 2011// J. Clin. Apher. - 2012 27; (3): 112-116.

[25] Clark WF, Huang SS, Walsh MW et al. Plasmapheresis for the treatment of kidney disease. Kidney Int 2018; 90 (5): 974-984.

[26] Wieliczko M, Dylewska M. IgA nephropathy - prognostic factors and treatment. Wiad Lek 2016; 69 (5): 707-710.

[27] Lechner SM, Papista C, Chemouny JM et al. Role of IgA receptors in the pathogenesis of IgA nephropathy. J Nephrol 2016; 29 (1): 5-11.

[28] Trimarchi H, Barratt J, Cattran DC et al. Oxford Classification of IgA nephropathy 2016: an update from the IgA Nephropathy Classification Working Group. Kidney Int 2017; 91 (5): 1014-1021. 28

[29] Stefan G, Ismail G, Stancu S et al. Validation study of Oxford Classification of IgA Nephropathy: the significance of extracapillary hypercellularity and mesangial $\operatorname{IgG}$ immunostaining. Pathol Int 2016; 66 (8): 453-459.

[30] Xie X, Ly J, Shi S et al. Plasma exchange as an adjuvant therapy for crescentic IgA nephropathy. Am J Nephrol 2016; 44 (2): 141-149.

[31] Saad M, Daoud M, Nasr P et al. IgA-dominant post-infectious glomerulonephritis presenting as a fatal pulmonary-renal syndrome. Int J Nephrol Renovasc Dis 2015; (8): 77-81.

[32] Yim DK, Lee ST, Cho H. Plasmapheresis therapy for pulmonary hemorrhage in a pediatric patients with $\operatorname{IgA}$ nephropathy. Korean J Pediatr 2015; 58 (10): 402-405.

[33] Kappler S, Ronan-Bentle S, Graham A. Thrombotic microangiopathies (TTP, HUS, HELLP). Emerg Med Clin North Am 2014; 32 (3): 649-671.

[34] Kremer Hovinga JA, Heeb SR, Skowronska M, Schaller M. Pathophysiology of thrombotic thrombocytopenic purpura and hemolytic uremic syndrome. J Tromb Haemost 2018; 16 (4.): 618-629.

[35] Coppo P, Veyradier A. Thrombotic microangiopathies: towards a pathophysiology-based classification. Cardiovasc Hematol Disord Drug Targets 2009; 9 (1): 36-50.

[36] Hidebrand AM, Huang SH, Clark WF. Plasma exchange for kidney disease: what is the best evidence? Adv Chronic Kidney Dis 2014; 21 (2): 217-227.

[37] Nakanishi T, Suzuki N, Kuragano T et al. Current topics in therapeutic plasmapheresis. Clin Exp Nephrol 2014; 18 (1): 41-49. 
[38] Martins I, Conceição MG, Gomes PP, Clode N. Hemolysis, elevated liver enzymes, low platelets syndrome superimposed on hemolytic uremic syndrome. Pev Bras Ginecol Obstet 2017; 39 (4): 195-198.

[39] Winiarska A, Kwella N, Stompór T. Heparin-unduced thrombocytopenia as a course of prolonged low platelet count in a patient with thrombotic thrombocytopenic purpura treated with plasmapheresis. Acta Biochim Pol 2017; 64 (2): 375-376.

[40] Sharma RK, Kaul A, Agrawal V, Jaisuresh K. Primapy antiphospholipid syndrome presenting as thrombotic microangiopathy: Successful treatment wiyh steroids, plasma exchange and anticoagulants. Indian J Nephrol 2011; 21 (4): 280-282.

[41] Ryu H, Kang E, Park S et al. A case of gemcitabine-induced thrombotic microangiopathy in a urogenital tumor patient with a single kidney. Kidney Res Clin Pract 2015; 34 (4): 237-340.

[42] Chen KR, Carlson JA Clinical approach to cutaneous vasculitis. Am J Clin Dermatol 2008; 9 (2): 71-92.

[43] Nissaisorakarn P, D’Agati V, Anis K, Jim B. ANCA and IgA glomerulonephritis all in one: prognosis and complications. BMJ Case Rep 2017; 2017: 222080.

[44] Jennette JC, Nachman PH. ANCA glomerulonephritis and vasculitis. Clin J Am Soc Nephrol 2017; 12 (11): P. 16801691.

[45] Popovic D, Pjeakovic S, Rankov O et al. Diabetic nephropathy in pregnant women with type 1 diabetes (multiple cases review and discussion. Georgian Med News 2018; 277: 15-20.

[46] Akhter F., Khan M. S., Alatar A. A. et al. Antigenic role of the adaptive immune response to d-ribose glycated LDL in diabetes, atherosclerosis and diabetes atherosclerotic patients. Life Sci 2016; 151: 139-146.

[47] Hickey FB, Martin F. Role of the immune system in diabetic kidney disease. Curr Diab Rep 2018; 18 (4): 20.

[48] Gul A, Zager P. Does altered uruc acid metabolism contribute to diabetic kidney disease pathophisiology. Curr Diab Rep 2018; 18 (4): 18.

[49] Sagoo MK, Gnudi L. Diabetic nephropathy: is there a role for oxidative stress? Free Radic Biol Med 2018; 116: 50-63.

[50] Bjornstad P, Cherney DZ. Renal hyperfiltration in adolescents with type 2 diabetes: physiology, sex differences, and implications for diabetic kidney disease. Curr Diab Rep 2018; $18(5) 22$

[51] Bus P, Chua JS, Lkessens CQF et al. Complement activation in patients with diabetic nephropathy. Kidney Int Rep 2017; 3 (2): 302-313.

[52] Lutfi R, Huang J, Wong HP. Plasmapheresis to treat hypertriglyceridemia in a child with diabetic ketoacidosis and pancreatitis. Pediatrics 2012; 129 (1): e195-198.

[53] Hardt U, Larsson A, Gunnarsson I et al. Autoimmune reactivity to malondialdehyde adducts in systemic lupus erythematosus is associated with disease activity and nephritis. Arthritis Res Ther 2018; 20 (1): 36.

[54] Li QY, Yu F, Zhou FD, Zhao MH. Plasmapheresis is associated with better renal outcomes in lupus nephritis patients with thrombotic microangiopathy: a case series study.
Medicine (Baltimore) 2016; 95 (18): e3595.

[55] Kronbichler A, Brezina B, Quintana LF, Jayne DR. Efficacy of plasma exchange and immunoadsorption in systemic lupus erythematosus and antiphospholipid syndrome: A systematic review. 2016; 15 (1): 38-49.

[56] Li M, Wang Y, Qiu Q et al. Therapeutic effect of doublefiltration plasmapheresis combined with methylprednisolone to treat diffuse proliferative lupus nephritis. J Clin Apher 2016; 31 (4): 375-380.

[57] Nakano T, Nagae H, Murakami $\mathrm{N}$ et al. Fever associated with severe dialysis- related amyloidosis. CEN Case Rep 2012; 1 (2): 112-116.

[58] Matsumoto K, Kikuchi J, Kaneko Y et al. Persistent fever and destructive arthritis caused by dialysis-related amyloidosis: a case report. Medicine (Baltimore) 2018; 97 (1): e9359.

[59] Joseph A, Harel S, Venot M et al. Renal recovery after severe acute kidney injury in critically ill myeloma patients: a retrospective study. Clin Kidney J 2018; 11 (1): 20-25.

[60] Scarpato S, Tirri E, Naclerio C et al. Plasmapheresis in cryoglobulinemic neuropathy: a clinical study. Dig Liver Dis 2007; 39, Suppl 1 (1) S136-137.

[61] Niaudet P. [Hemolytic and uremic syndrome in the child]. Nephrol Ther 2008; 4 (1): 34-40 (Fr).

[62] Amirlak I, Amirlak B. Haemolytic uraemic syndrome: an overview. Nephrology (Carlton) 2006; 11 (3): 213-218.

[63] Van Gemert LM, Montemayor-Garcia C, Rose WN. Dramatic improvement immediately after plasma exchange in a patient with Shiga toxin-producing Escherichia Coli hemolytic uremic syndrome. J Clin Apher 2014; 29 (1): 46.

[64] Loos S, Aulbert W, Hoppe B et al. Intermediat follow-up of pediatric patients with hemolytic uremic syndrome during the 2011 outbreak caused by E. coli O104:H4. Clin Infect Dis 2017; 64 (122): 1637-1643.

[65] Kielstein JT, Beutel G, Fieig S et al. Best supportive care and therapeutic plasma exchange with or without eculizumab in Shiga-toxin-producing E. coli O104:H4 induced haemolytic-uraemic syndrome: an analysis of the German STEC-HUS registry. Nephrol Dial Transplant 2012: 27 (10): 3807-3815.

[66] Ozkok A, Yildriz A. Hepatitis C virus associated glomerulopathies. World J Gastroenterol 2014; 20 (24): 7544 7554.

[67] Balwani MR, Kute VB, Shah PR et al. Hepatitis B viremia manifesting as polyarteritis nodosa and secondary membranous nephropathy. J Nephropharmacol 2016; 5 (2): 119-121.

[68] Mareddy AS, Rangaswamy D, Vankalakunti M et al. Immune mediated crescentic MPGN secondary to HBV infection: A rare presentation for common infection. Australas Med J 2016; 31 (1): $12-16$

[69] Narayanan M, Casimiro I, Pichler R. A unique way to treat Goodpasture's disease. BMJ Case Rep 2014; Nov 24: 2014206220.

[70] Milovanov Yu S [Plasmapheresis in nephrology]. In: "Clinical application of extracorporeal treatments." Moscow, 2006: 7275 (Rus). 
[71] Okuyama H, Kimura S, Fujimoto K et al. A case of chronic hepatitis $\mathrm{C}$ with nephrotic diabetic nephropathy who achived sustained viral remission by double-filtration plasmapheresis and interferon combination therapy. Intern Med 2012; 51: 1991-1995.

[72] Shelukhin, VA, Kostyuchenko AL. [Apheresis therapy in diseases and lesions of the kidneys]. In: Efferent therapy/ edited by AL Kostyuchenko. St. Petersburg: Folio 2003: 268302 (Rus).

[73] Dovlatyan A. [Intensive care of complicated forms of purulent gestational pyelonephritis]. Urology 2008; 2: 10-14 (Rus).

[74] Akhmedzhanova ZM, Vetrov VV, Konovalova MV, Taits AN. [Plasmapheresis and photomodification of blood in prevention of septic complications in puerperas]. Proc. Confer. "Therapeutic Hemapheresis" St. Petersburg 2017: 14-15. (Rus).

[75] Neymark AI, Yakovets YaV, Astakhov YuI. [Plasmapheresis in correction of violations of a hemostasis system at patients with acute and chronic pyelonephritis]. Efferent Therapy (Rus) 1996; 2 (4): 45-49.

[76] Crumley S, Ge Y, Zhou H et al. Interstitial cystitis: another IgG4-related inflammatory disease? Ann Diagn Pathol 2013; 17 (5): 403-407.

[77] Lee J, Doggweiler-Wiygul R, Kim S et al. Is interstitial cystitis an allergic disorder? A case of cystitis treated successfully with anti-IgE. Int J Urol 2006; 13 (5): 631-634.

[78] Boissier E, Durant C, Vildy S et al. An unexpected etiology of priapism: infection-related anti-proteins antibodies. J Sex Med 2014; 11 (11): 2830-2833.

[79] Restrepo B, Cardona-Maya W. Antisperm antibodies and fertility association. Actas Urol Esp 2013; 37 (9): 571-578.

[80] Tchiokadze Sh, Galdava G Clinical and anamnestic characteristics of development of antisperm immunity in infertile men. Georgian Med News 2015; 246: 18-22.

[81] Drobnis EZ, Nangia AK. Immunosuppressants and male reproduction. Adv Exp Med Biol 2017; 1034: 179-210.

[82] Benedetti S, Tagliamonte MC, Catalani S et al. Differences in blood and semen oxidative status in fertile and infertile men, and their relationship with sperm quality. Reprod Biomed Online 2012; 25 (3): 300-306.

[83] Agarwal A, Durairajanayagam D, Halabi J et al. Proteomics, oxidative stress and male infertility. Reprod Biomed Online 2014; 29 (1): 32-58.

[84] Ambulkar PS, Chuadhari AR, Pal AK. Association of large scale 4977-bp "common" deletions in sperm mitochondrial DNA with asthenozoospermia and oligoasthenoteratozoospermia. J Hum Reprod Sci 2016; 9 (1): 35-40.

[85] Korenkov DG, Alexandrov VP, Marusanov VE et al. [Features of application of efferent methods at correction of immunodependent infertility at men. Efferent Therapy (Rus) 2003; 9 (2): 43-49.

[86] Atyushev GP, Motavkina NS. [Viral infections in genesis of antispermal immunity and infertility at patients with urogenital infections, sexually transmitted]. Allergol Immunol (Rus) 2006; 7 (3): 391-392.

[87] Tiseo BC, Cocuzza M, Bonfa F et al. Male fertility potential alteration in rheumatic diseases: a systematic review. Int Braz J Urol 2016; 42 (1): 11-21.

[88] Dupree JM. Insuarance coverage for male infertility care in the United States// Asian J Androl 2016; 18 (3): 339-341.

[89] Andrievskikh IA, Fokin AA, Grafov AA. [Effect of immunocorrection on surgical outcomes in patients with autoimmune-aetiology vasculitis and vasculopathies]. Angiol Sosud Khir (Rus) 2008; 14 (2): 20-26.

[90] Gungor O, Sen S, Kircelli F et al. Plasmapheresis therapy in renal transplant patients: five-year experience. Transplant Proc 2011; 43: 853-857.

[91] Yazici O, Sendur MA, Aksoy S. Hepatitis C virus reactivation in cancer patients in the era of targeted therapies. World $\mathrm{J}$ Gastroenterol 2014; 20 (22): 6716-6724.

[92] Abbas A, Mitza MM, Ganti AK, Tendulkar K. Renal toxicities of targeted therapies. Target Oncol 2015; 10 (4): 487-499.

[93] Okuda $\mathrm{Y}$, Ishikura $\mathrm{K}$, Terano $\mathrm{C}$ et al. Irreversible severe kidney injury and anuria in a 3-month-old girl with atypical haemolytic uraemic syndrome under administration of eculizumab. Nephrology (Carlton) 2016; 21 (3): 261-265.

[94] Masmoudi A, Hajiaji Darouiche M, Ben Salah H et al. Cutaneous abnormalities in patients with end stage renal failure on chronic hemodialysis. A study of 458 patients. J Dermatol Case Rep 2014; 8: 86-94.

[95] Fuhrmann V., Drilz A., Trauner M. Extracorporeal artificial liver support systems in the management of intractable cholestatic pruritus. Liver Int 2011; 31 (Suppl. 3): 31-33.

[96] Dechmann-Sultemeyer T, Linkeschova R, Lenztn $\mathrm{K}$ et al. Tandem plasmapheresis and haemodialisis as a safe procedure in 82 patients with immune-mediated disease. Nephrol Dial Transplant 2009; 24 (1): 252-257.

[97] Loo CY, Mohamed Said MS, Mohd R et al. Immunoadsorption and plasmapheresis are equally efficacious as adjunctive therapies for severe lupus nephritis. Transfus Apher Sci 2010; 43 (3): 335-340.

[98] Voinov VA. Therapeutic apheresis. Constanţa: Celebris. 2016: $400 \mathrm{p}$.

[99] Voinov VA, Karchevsky KS, Isaulov OV. [Plasmapheresis in nephrology]. Nephrologia (Rus) 2017; Vol. 21 (4): 95-101. 